\title{
Exceptional Cause of Respiratory Distress: Bilateral Congenital Lobar Emphysema
}

\author{
Ait Chtouk M*, El Badri Y, Zouita B, Basraoui D, Jalal H
}

Radiology Department, child hospital, CHU Med VI, Marrakesh, Morocco

DOI: $10.36347 /$ sjmcr.2020.v08i04.023

| Received: 06.03.2020 | Accepted: 13.03.2020 | Published: 30.04 .2020

*Corresponding author: Ait Chtouk Mohamed

Abstract

We report a case of a 3 months old male who presented with increasing respiratory distress since birth aggravated two weeks before admission and was diagnosed to have bilateral congenital lobar emphysema on CT.

Keywords: Bilateral congenital lobar emphysema, CT.

Copyright @ 2020: This is an open-access article distributed under the terms of the Creative Commons Attribution license which permits unrestricted use, distribution, and reproduction in any medium for non-commercial use (NonCommercial, or CC-BY-NC) provided the original author and source are credited.

\section{INTRODUCTION}

Congenital lobar emphysema (CLE) is a rare anomaly of lung development which is usually unilateral [1]. Bilateral involvement is exceptionally rare and has been reported only in few cases in the literature [2-4]. CLE usually results in progressive respiratory distress in early infancy [1]; We report a case of bilateral CLE in a 3-month-old male infant presented with acute respiratory distress. Our case report and literature review highlight the management difficulties in our understanding of this difficult and rare malformation.

\section{CASE Report}

A 3-month-old male infant was admitted for respiratory distress, wheezing and failure to thrive. Personal physiological history showed that he was from a physiological pregnancy, born at term by C-section, with a birth weight of $2950 \mathrm{~g}$, an Apgar score was 9 and 10 at 1 and 5 minutes, respectively, and a normal psychosomatic development. Family history was not significant, and personal pathological history included a congenital infection of undetermined etiology.

The clinical onset of the disease was two months before admission, by installing a cough, wheezing, and progressive respiratory distress but no fever, but evolution showed aggravation of dyspnoea requiring hospitalization.

On admission, he was found to be tachypneic at a rate of $68-70 / \mathrm{min}$, pulse rate of $169 / \mathrm{min}$, and blood pressure of $84 / 50 \mathrm{mmHg}$. His temperature was $36.4^{\circ} \mathrm{C}$ and his $\mathrm{O} 2$ saturation was 92 to $94 \%$ on room air. Chest examination revealed subcostal and intercostal retractions, decreased air entry in both sides with expiratory wheeze bilaterally. A facial dysmorphism has been found; Rest of systemic examination including cardiovascular examination was within normal. Infant was supplemented with oxygen, intravenous fluids, and a trial of inhaled bronchodilator. Respiratory status worsened significantly and infant was transferred to pediatric resuscitation where he was intubated and mechanically ventilated.

Chest X-ray showed middle lobe and the upper $2 / 3$ of the left pulmonary hemichamps hyperinflation with widening intercostal spaces (Figure $1)$.

A echocardiography reveal atrial septal defect. Chest computed tomography detected distention with hyperinflation associated, rarefaction and small aspect of the vascular structures in the middle lobe and the left upper lobe causing mediastinal displacement to the left side (Figure 2) 


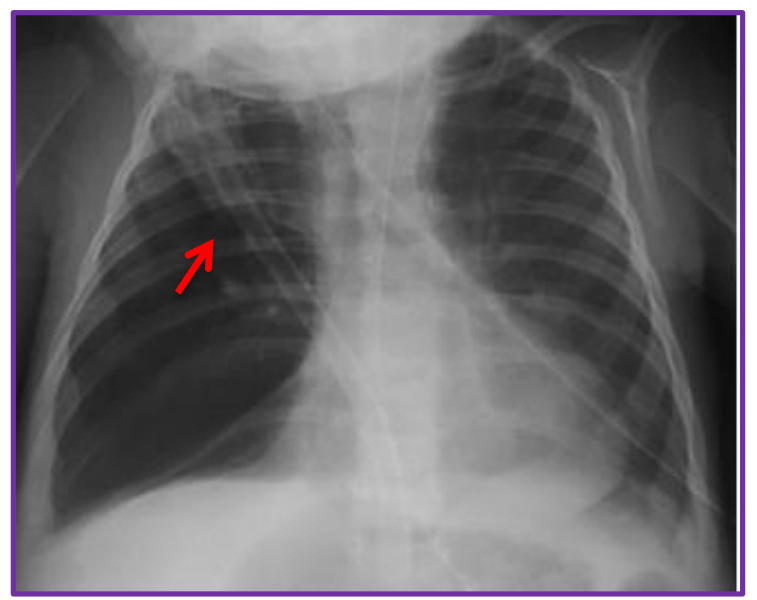

Fig-1: Chest x-ray: hyperinflated in the middle lobe (limited at the top by the small caesura red arrow) the upper $2 / 3$ hemi left pulmonary field, with widening intercostal spaces, horizontalization of the ribs and pulmonary hypovascularization.

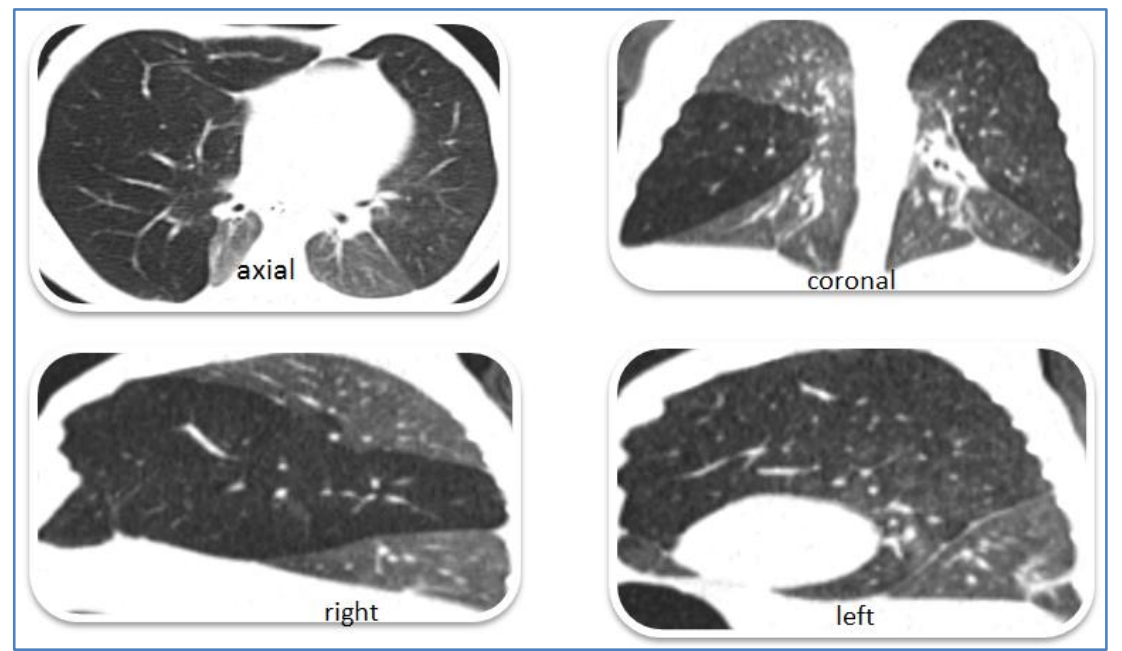

Fig-2: Chest computed tomography in parenchymal window: Distention with hyperinflation associated, rarefaction and small aspect of the vascular structures in the middle lobe and the left upper lobe causing mediastinal displacement to the left side

\section{DiscUSSION}

Congenital lobar emphysema is an uncommon childhood respiratory tract disease characterized by overinflation of a pulmonary lobe. The exact cause of congenital lobar emphysema is difficult to determine and seems even non-existent in $50 \%$ of cases and can be attributed to a congenital bronchial cartilage defect, extrinsic compression of aberrant vessels, bronchial stenosis, viscous bronchial mucus, and mediastinal displacement to the opposite side of bronchial obstruction [5]. Our patient had a normal vascular anatomy and bronchial cartilage on gross examination nor were there any other lung abnormalities; In 10\% of cases, congenital lobar emphysema can be associated with congenital cardiac malformations [6]; Our patients had atrial septal defect confirmed by echocardiography.

Bilobar or multifocal involvement is extremely rare [7].Bilateral localization was more often reported than unilateral localization. The most commonly affected parts of the lungs are the LUL and RML [3, 8, 9]; In our case report, bilobar emphysema was bilatéral.
The age of onset of symptoms of congenital lobar emphysema ranges from a few days after birth to six months [9]; Most patients develop symptoms in the neonatal period and the male: female ratio is $3: 1$ [10]; As in this child, respiratory distress is the commonest mode of presentation. There is dyspnoea, wheezing, grunting respiration, tachypnoea and sometimes progressive cyanosis. Similar symptoms may occur in bronchopneumonia, cyanotic congenital heart diseases, and several congenital abnormalities of the lung [11].

Antenatal detection of CLE and other lung anomalies can be done at midgestation using ultrasonography and fetal MRI which was missed in our case.

The basic investigation in congenital lobar emphysema is the chest radiograph wich often showed a hyperinflated hemithorax and mediastinal shift to the contralateral site. In the case presenting with a large emphysematous lobe, lung herniation to the contralateral hemithorax and atelectasis of the contralateral lung can be seen. 
The CT scanning of the chest may reveal bilobar involvement and may show bronchial obstruction. It may have negative results in patients with bilobar emphysema as demonstrated in case 1 because of mediastinal shift, atelectasis, or compressed near lobe [12]. Perfusion scintigraphy can show decreased perfusion secondary to the compression of surrounding blood vessels. It is more sensitive for bilobar involvement and seems appropriate to evaluate cases of congenital lobar emphysema for the possibility of multifocality. Radionuclide ventilation/perfusion scintigraphy is suggested as a complementary imaging method before initial treatment [13].

Surgical approach of bilateral CLE is controversial. Some reports are advocating the two-step sequential resection of the emphysematous lobes [2, 8], while others support the one-step operation in which the affected lobes are excised simultaneously [3, 4]. Our patient died after the treatment was established by worsening his dyspnoea.

\section{CONCLUSION}

Bilateral CLE is a rare condition, and detailed work-up of these patients is important. Depending on the severity and lobar involvement, it may be managed as a single stage or as a two-staged procedure, as in our case. Surgical management should be guided by clinical and radiological evaluation after excision of the most severely affected lobe.

\section{REFERENCES}

1. Abel RM, Bush A. Congenital Lung Disease. In: Victor Chernick, Editor. Kendig's disorders of the respiratory tract in children. 7th Ed. Philadelphia. Saudners; 2006. p. 280-316.

2. Floyd FW, Repici AJ, Gibson ET, Mcgeorge CK. Bilateral Congenital labor emphysema surgically corrected. Pediatrics. 1963;1:87-96.
3. Ekkelkamp S, Vos A. Successful surgical treatment of a newborn with bilateral congenital lobar emphysema. J Pediatr Surg. 1987;22:1001-2.

4. Iodice F, Harban F, Walker I. Anesthetic management of a patient with bilateral congenital lobar emphysema. Paediatr Anaesth. 2008;18:3401

5. Center for fetal Diagnosis and Treatment - The Children's Hospital of Philadelphia, PA, USA. Prenatal diagnosis and management of congenital lobar emphysema. J Pediatr Surg. 2000;35:792795.

6. Prabhu M, Joseph TT. Congenital lobar emphysema: Challanges in diagnosis and ventilation. Anesth Essays Res. 2012;6:203-206.

7. Hugosson C, Rabeeah A, Al-Rawaf. Congenital bilobar emphysema. Pediatr Radiol 1995;25:649-51

8. Maiya S, Clarke JR, More B. Bilateral congenital lobar emphysema: how should we proceed? Pediatr Surg Int. 2005;21:659-61.

9. Bappal B, Ghani SA, Chaudhary R, Sajvani MJ. Congenital lobar emphysema: a review of 10 cases. Indian- J-Pediatr. 1996;63(6):801-808

10. Cay A, Sarihan H. Congenital malformations of the lung. J-Cardiovasc-Sur-(Adam). 2000;41(3):507510.

11. Takeda S, Miyoshi S, Inoue M, Omori K, Okumura M, Yoon HE, Minami M, Matsuda H. Clinical spectrum of congenital cystic disease of the lung in children. Eur-J-Cardiothorac-Surg. 1999;15(1):11 17.

12. Mikhailova V. Congenital lobar emphysema in childhood. Khirurgiia (Sofia). 1996;49(3):8-12.

13. Absher DR, Kriss VM, Cotrill CM. Lobar emphysema due to anomalous aortic origin of theleft pulmonary artery. CardiolYoung. 1999;9(3):327-330. 